\title{
Dupilumab for Chronic Eosinophilic Pneumonia
}

\author{
Christopher Fowler ${ }^{1}$ and Wynton Hoover ${ }^{2}$ \\ ${ }^{1}$ The University of Alabama at Birmingham \\ 2Indiana University
}

July 7, 2020

\begin{abstract}
Chronic Eosinophilic Pneumonia (CEP) is a rare idiopathic eosinophilic lung disease characterized by a triad of pulmonary symptoms, radiographic findings and peripheral and/or lung eosinophilia. CEP is usually treated with oral corticosteroids however, treatment durations commonly exceed 12 months, relapses occur in $\frac{1}{2}$ of patients and adverse effects of steroid use are common. We report a case of an 11-year-old female with corticosteroid refractory CEP who experienced clinical remission after initiation of dupilumab, a monoclonal antibody therapy approved for eosinophilic or steroid dependent asthma that inhibits IL-4 and IL-13. This case demonstrates the potential role of biologic agents in CEP.
\end{abstract}

\section{Text}

Introduction

Chronic eosinophilic pneumonia (CEP) is an idiopathic disease rarely reported in children ${ }^{1,2}$. It typically occurs in non-smoking, middle age females and the diagnosis requires exclusion of systemic or primary eosinophilic lung diseases ${ }^{1,2}$. The triad of pulmonary symptoms (usually for $>2$ weeks), abnormal chest radiographic findings and eosinophilia in the blood and/or pulmonary tissue in the absence of an alternative etiology is highly suggestive of CEP. Patients may present with cough (90-93\%) dyspnea (50-90\%), fever (77$87 \%$ ), night sweats (23\%), weight loss (57-75\%), rhinitis or sinusitis (6-24\%), chest pain (8-16\%), hemoptysis $(8-10 \%)$, and weakness/fatigue ${ }^{1,2}$. An asthma phenotype is present in roughly a third to $3 / 4$ of patients. Bilateral peripheral consolidative infiltrates are seen on chest radiograph (CXR) with computed tomography (CT) showing peripheral airspace disease and reactive hilar adenopathy ${ }^{1,2}$. Peripheral eosinophilia is almost universal with cell counts routinely higher than $1000 / \mathrm{mm}^{3}$ and more than half of patients also have an elevated $\operatorname{IgE}^{2}$. Eosinophilia on the bronchoalveolar lavage (BAL) ranges from 12 to $95 \%$ with a mean of $58 \%^{2}$.

The triad of pulmonary symptoms, radiographic findings and eosinophilia in CEP typically respond to prednisone within a few days ${ }^{2}$. Initial doses range from 0.5 to $1 \mathrm{mg} / \mathrm{kg} /$ day and may be tapered after clini$\mathrm{cal} /$ radiographic improvement. Duration of therapy depends on the response and is highly variable, with a mean of 19 months but a range of 0.5 to 96 months $^{1}$. Relapse is common, occurring in up $50 \%$ of patients ${ }^{2}$.

While the pathogenesis of CEP is not fully understood, the underlying mechanism undoubtedly involves eosinophils and likely shares inflammatory pathways with asthma, which is why it may be amenable to treatment with novel biologic therapies approved for asthma. Although there are case reports of patients with CEP who were treated with either omalizumab or mepolizumab, there are no reports of treatment with dupilumab ${ }^{3}$. However, there is a report of a patient possibly experiencing CEP as a consequence of dupilumab ${ }^{4}$.

Case Report 
An 11-year-old, previously healthy, African American female presented with fever, cough, dyspnea, chest pain and decreased appetite of 3 months duration. Her past medical history was significant for pneumonia three months prior that failed to resolve with antibiotics. She was subsequently prescribed albuterol and treated as an outpatient with a working diagnosis of asthma. Her symptoms persisted and she was ultimately hospitalized with chronic pneumonia of unclear etiology. Her family and social histories were unremarkable. She strongly denied vaping or inhalant abuse. Her physical exam was remarkable for bilateral coarse crackles, signs of respiratory distress and reproducible chest pain. Her CXR showed bilateral consolidative opacities and her CT demonstrated bilateral lymphadenopathy and multilobar consolidation (see Figures $1 \& 2$ ). Her initial lab work was remarkable for a white blood cell count of 10,000/ $\mu \mathrm{L}(3.84-9.84 / \mu \mathrm{L})$ with $10 \%(0-4 \%)$ peripheral eosinophilia, erythrocyte sedimentation rate of $103 \mathrm{~mm} / \mathrm{hr}(0-15 \mathrm{~mm} / \mathrm{hr})$, and C reactive protein of $7 \mathrm{mg} / \mathrm{dL}(0.00-0.50 \mathrm{mg} / \mathrm{dL})$. She was treated with 14 days of antibiotics without clinical improvement. During the course of follow up her FEV1 declined $>35 \%$ and she developed a severe persistent asthma phenotype. She was evaluated for tuberculosis, allergic bronchopulmonary aspergillosis, helminth infection, eosinophilic granulomatosis with polyangitis, sarcoidosis, and immunodeficiency however her work up was negative. She underwent bronchoscopy, which demonstrated significant bilateral lower airway casts that were $93 \%$ eosinophilic. She was treated with high dose prednisone for 4 weeks and improved for a few months, however her symptoms returned. She continued oral prednisone intermittently for approximately 22 months without improvement and was deemed refractory to therapy. Repeat imaging during that time demonstrated persistent opacities and she developed bronchiectasis. Repeat bronchoscopies revealed return of mucoid impactions. Throughout her course, she continued to have eosinophilia in the blood (ranging from 0.4 to $13 \%$ ) and on BAL (ranging from 9-93\%).

She was then treated with pulse methylprednisolone and daily cyclosporine in an attempt to gain clinical remission which resulted in modest improvement of symptoms. She continued daily cyclosporine with the addition of dupilumab every 2 weeks for 6 months. Following the addition of dupilumab significant clinical and radiographic improvement were noted within 2 weeks. Her cyclosporine was subsequently weaned without recurrence of symptoms and she remains symptom free with marked improvement in her Chest X-ray findings for over 12 months on subcutaneous dupilumab injections q 2 weeks.

Discussion

Dupilumab is an injectable humanized IgG4 monoclonal antibody, approved for home use in patients with eosinophilic or steroid dependent asthma, which targets IL- $4 \alpha$ receptors and inhibits IL- 4 and IL- 13 binding. It has been shown to reduce the frequency of asthma exacerbations, improve FEV1 and reduce oral steroid use in patients with severe asthma ${ }^{5}$. This is the first report of dupilumab as a therapy to treat CEP and the first report of monoclonal antibody therapy to treat CEP in a pediatric patient. Dupilumab may be an alternative for CEP that is refractory to corticosteroids or that requires prolonged therapy. It may also be a more convenient therapy compared to other monoclonal antibodies available to patients because it can be administered at home.

\section{References}

1. Jederlinic PJ, Sicilian L, Gaensler EA. 1988. Chronic eosinophilic pneumonia. A report of 19 cases and a review of the literature. Medicine (Baltimore). 67(3):154-162.

2. Marchand E, Reynaud-Gaubert M, Lauque D, Durieu J, Tonnel AB, Cordier JF. 1998. Idiopathic chronic eosinophilic pneumonia. A clinical and follow-up study of 62 cases. The groupe d'etudes et de recherche sur les maladies "orphelines" pulmonaires (germ"o"p). Medicine (Baltimore). 77(5):299-312.

3. Lin RY, Santiago TP, Patel NM. 2019. Favorable response to asthma-dosed subcutaneous mepolizumab in eosinophilic pneumonia. J Asthma. 56(11):1193-1197.

4. Menzella F, Montanari G, Patricelli G, Cavazza A, Galeone C, Ruggiero P, Bagnasco D, Facciolongo N. 2019. A case of chronic eosinophilic pneumonia in a patient treated with dupilumab. Ther Clin Risk Manag. 15:869-875. 
5. Castro M, Rabe KF, Corren J, Pavord ID, Katelaris CH, Tohda Y, Zhang B, Rice MS, Maroni J, Rowe $\mathrm{P}$ et al. 2020. Dupilumab improves lung function in patients with uncontrolled, moderate-to-severe asthma. ERJ Open Res. 6(1).
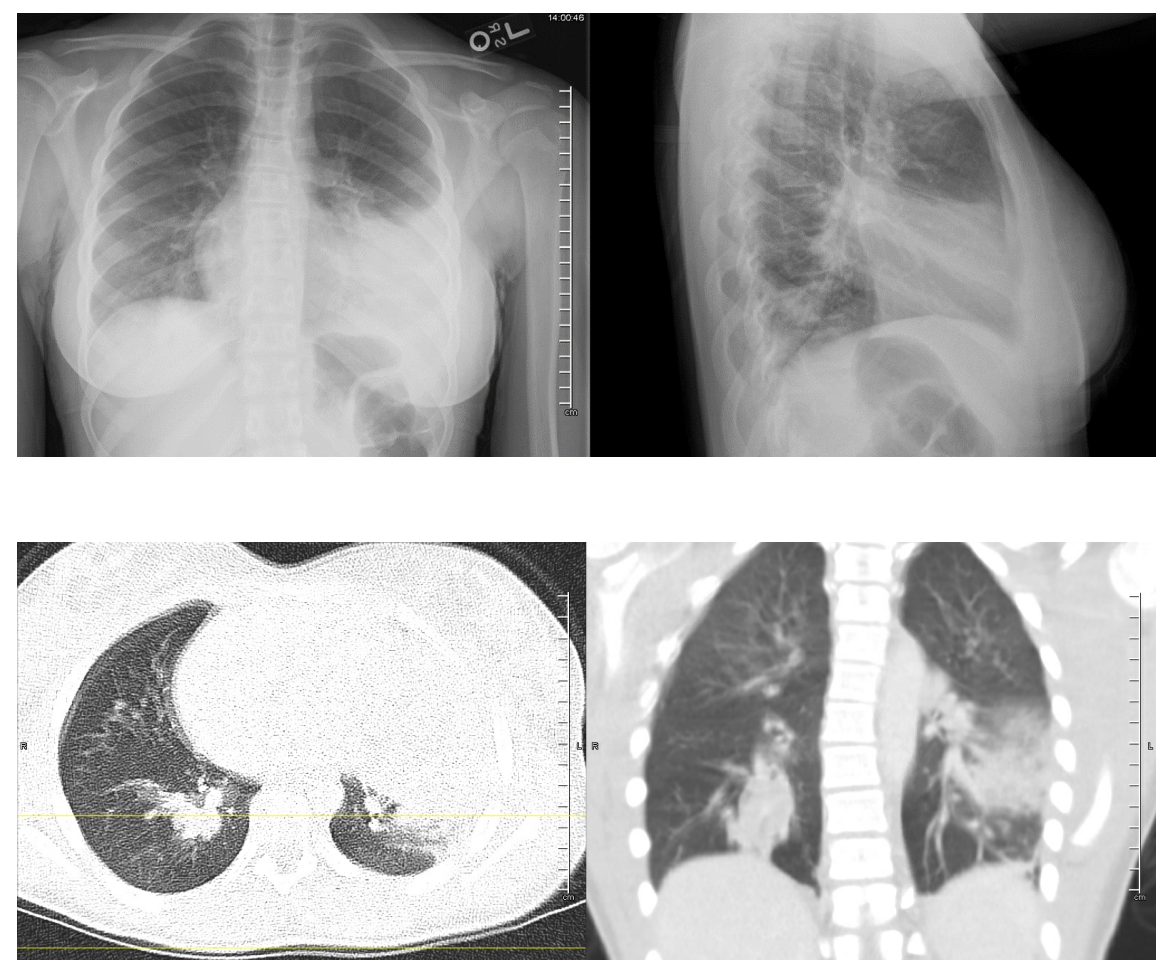